\title{
WAVES AND WAVE FORCES ON COASTAL AND OCEAN STRUCTURES
}


Series Editor-in-Chief

Philip L-F Liu (Cornell University)

Vol. 9 Offshore Structure Modeling

by Subrata K. Chakrabarti (Chicago Bridge \& Iron Technical

Services Co., USA)

Vol. 10 Water Waves Generated by Underwater Explosion

by Bernard Le Méhauté and Shen Wang (Univ. Miami)

Vol. 11 Ocean Surface Waves; Their Physics and Prediction by Stanislaw $R$ Massel (Australian Inst. of Marine Sci)

Vol. 12 Hydrodynamics Around Cylindrical Structures

by $B$ Mutlu Sumer and Jørgen Fredsøe (Tech. Univ. of Denmark)

Vol. 13 Water Wave Propagation Over Uneven Bottoms

Part I - Linear Wave Propagation

by Maarten W Dingemans (Delft Hydraulics)

Part II - Non-linear Wave Propagation

by Maarten W Dingemans (Delft Hydraulics)

Vol. 14 Coastal Stabilization

by Richard Silvester and John R C Hsu (The Univ. of Western Australia)

Vol. 15 Random Seas and Design of Maritime Structures (2nd Edition)

by Yoshimi Goda (Yokohama National University)

Vol. 16 introduction to Coastal Engineering and Management

by $J$ William Kamphuis (Queen's Univ.)

Vol. 17 The Mechanics of Scour in the Marine Environment by $B$ Mutlu Sumer and Jørgen Fredsøe (Tech. Univ. of Denmark)

Vol. 18 Beach Nourishment: Theory and Practice by Robert G. Dean (Univ. Florida)

Vol. 19 Saving America's Beaches: The Causes of and Solutions to Beach Erosion by Scott L. Douglass (Univ. South Alabama)

Vol. 20 The Theory and Practice of Hydrodynamics and Vibration by Subrata K. Chakrabarti (Offshore Structure Analysis, Inc., Illinois, USA)

Vol. 21 Waves and Wave Forces on Coastal and Ocean Structures by Robert T. Hudspeth (Oregon State Univ., USA)

Vol. 22 The Dynamics of Marine Craft: Maneuvering and Seakeeping by Edward M. Lewandowski (Computer Sciences Corporation, USA)

Vol. 23 Theory and Applications of Ocean Surface Waves

Part 1: Linear Aspects

Part 2: Nonlinear Aspects

by Chiang C. Mei (Massachusetts Inst. of Technology, USA),

Michael Stiassnie (Technion-Israel Inst. of Technology, Israel) and

Dick K. P. Yue (Massachusetts Inst. of Technology, USA)

Vol. 24 Introduction to Nearshore Hydrodynamics

by $l b$ A. Svendsen (Univ. of Delaware, USA) 
Advanced Series on Ocean Engineering - Volume 21

\section{WAVES AND WAVE FORCES ON COASTAL AND OCEAN STRUCTURES}

\section{Robert T. Hudspeth}

Oregon State University, USA

\section{World Scientific}




\section{Published by}

World Scientific Publishing Co. Pte. Ltd.

5 Toh Tuck Link, Singapore 596224

USA office: 27 Warren Street, Suite 401-402, Hackensack, NJ 07601

UK office: 57 Shelton Street, Covent Garden, London WC2H 9HE

\section{British Library Cataloguing-in-Publication Data}

A catalogue record for this book is available from the British Library.

\section{WAVES AND WAVE FORCES ON COASTAL AND OCEAN STRUCTURES}

Copyright $\mathbb{C} 2006$ by World Scientific Publishing Co. Pte. Ltd.

All rights reserved. This book, or parts thereof, may not be reproduced in any form or by any means, electronic or mechanical, including photocopying, recording or any information storage and retrieval system now known or to be invented, without written permission from the Publisher.

For photocopying of material in this volume, please pay a copying fee through the Copyright Clearance Center, Inc., 222 Rosewood Drive, Danvers, MA 01923, USA. In this case permission to photocopy is not required from the publisher.

ISBN 981-238-612-2

Typeset by Stallion Press

Email: enquiries@stallionpress.com

Printed in Singapore by B \& JO Enterprise 


\section{a Charo y Roberto \\ ¡ los tesoros de mi vida!}


This page is intentionally left blank 


\section{Contents}

Preface $\quad x v$

1 Introduction 1

2 Mathematical Preliminaries 5

2.1 Introduction 5

2.2 Symbols, Functions and Linear Operators 5

2.2.1 Landau Order Symbols $O(\epsilon)$ and $o(\epsilon)$ (Nayfeh, 1973, Chapter 1.3 and Olver, 1990, Chapter 12.1.1) 5

2.2.2 Heaviside Step Function $U(x-\xi) \quad 7$

2.2.3 Kronecker Delta $\delta_{m n}$ Function and Dirac Delta $\delta(x-\xi)$ Distribution 7

2.2.4 Levi-Civita Symbol $\varepsilon_{i j k}$ (Arfken, 1985) 7

2.2.5 Gamma Functions $\Gamma(\bullet)$ (Andrews, 1985) 8

2.2.6 Error Functions $\operatorname{Erf}(\bullet)$ and $\operatorname{Erfc}(\bullet)$ (Barcilon, 1990, p. 351) 9

2.2.7 Gradient Vector Operator $\vec{\nabla}(\bullet) \quad 10$

2.2.8 Curl Vector Operator $\vec{w}=\vec{\nabla} \times(\vec{\bullet}) \quad 10$

2.2.9 Laplacian Operator $\nabla^{2}(\bullet)=\Delta(\bullet)$

2.2.10 Stokes Material Derivative Operator $D(\bullet) / D t \quad 11$

2.2.11 Leibnitz's Rule for Differentiation of Integrals with
Parameters (Hildebrand, 1976, Chapter 7.9)

2.2.12 Signum (sign \pm ) Function 13 
2.3 Properties of Series

2.3.1 Power Series (Hildebrand, 1976, Chapter 4.1) 13

2.3.2 Function Series

2.3.3 Maclauren and Taylor Series (Hildebrand, 1976, Chapters 4.1 and 7.5)

2.3.4 Binomial Expansion (Wylie and Barrett, 1982, p. 938)

2.4 Elementary and Special Functions (Hildebrand, 1976, Chapter 10.2)

2.4.1 Trigonometric and Hyperbolic Identities

2.4.2 Euler's Constant $\gamma_{E}$ (Barcilon, 1990, p. 346)

2.4.3 Bessel Functions (Hildebrand, 1976, Chapters 4.8 to 4.10 )

2.4.4 Orthogonal Polynomials

2.5 Linear Ordinary Differential Equations (Hildebrand, 1976, Chapters 1.1 to 1.11 ) and Operational Calculus

(Friedman, 1956)

2.5.1 Initial and Boundary Data (Stakgold, 1979)

2.5.2 First Order Linear Ordinary Differential Equations (Hildebrand, 1976, Chapter 1.4)

2.5.3 Variation of Parameters and the Duhamel Convolution Integral (Hildebrand, 1976, Chapter 1.9)

2.5.4 Properties of Linear Differential Operators $\mathcal{L}(\bullet)$ (Hildebrand, 1976, Chapter 1.7)

2.5.5 Method of Frobenius (Hildebrand, 1976, Chapter 4.4)

2.5.6 Method of Undetermined Coefficients (Hildebrand, 1976, Chapter 1.5)

2.6 Sturm-Liouville Systems (Morse and Feshbach, 1953, Chapter 6.3; Hildebrand, 1976, Chapter 5.6; Oates, 1990, Chapter 3.6.5 and Benton, 1990, Chapter 6.6)

3 Fundamentals of Fluid Mechanics

3.1 Introduction

3.2 Conservation of Mass (Continuity Field Equation) 
3.3 Momentum Principle $\quad 57$

3.3.1 Inertial Forces $\quad 58$

3.3.2 Surface Stresses $\quad 63$

3.3.3 Body Forces 69

3.3.4 Navier-Stokes Equations (Lamb, 1932) 69

3.3.5 Euler's Equations (Lamb, 1932) 70

3.4 Mechanical Energy Principle $\quad 70$

$\begin{array}{ll}3.5 & \text { Scaling of Equations }\end{array}$

3.6 Dimensional Analyses $\quad 81$

3.7 Problems $\quad 84$

4 Long-Crested, Linear Wave Theory (LWT) 85

4.1 Introduction 85

4.2 Dimensional Boundary Value Problem (BVP) for LWT 87

4.3 Solutions to Dimensional Boundary Value Problem (BVP) for Long-Crested, Linear Wave Theory (LWT) 97

4.3.1 Wave Celerity $C$ and Computing the Eigenvalue $k$ from the Frequency Dispersion Equation

4.4 Eulerian Kinematic Fields and Lagrangian

Particle Displacements

4.5 Eulerian Dynamic Fields, Energy and Energy Flux

Conservation Principles for Long-Crested Linear Waves

4.6 Wave Transformations for Long-Crested, Progressive Linear

Waves: Shoaling and Refraction

$\begin{array}{lll}4.7 & \text { Problems } & 144\end{array}$

5 Wavemaker Theories 149

$\begin{array}{lll}5.1 \text { Introduction } & 149\end{array}$

5.2 Planar Wavemakers in a 2D Channel 154

5.2.1 Computation of the Eigenvalues $K_{n}$ by the Newton-Raphson Method 170

5.2.2 Rate of Decay of Evanescent Eigenmodes: $n \geq 2$

5.2.3 Orthogonality of Orthonormal Eigenfunctions $\Psi_{n}\left(K_{n}, z / h\right)$ 
5.2.4 Evaluation of Coefficients $C_{n}$ by Wavemaker Vertical Displacement $\chi(z / h)$

5.2.5 Determination of Wave Amplitude from Wavemaker Motion

5.2.6 Hydrodynamic Pressure Force and Moment (Added Mass and Radiation Damping)

5.3 Circular Wavemakers

5.3.1 Determination of Wave Amplitude from Wavemaker Motion

5.3.2 Hydrodynamic Pressure Force and Moment (Added Mass and Radiation Damping)

5.4 Double-Actuated Wavemaker

5.5 Directional Wavemaker

5.6 Sloshing Waves in a 2D Wave Channel

$\begin{array}{ll}5.7 \text { Conformal and Domain Mapping of WMBVP } & 279\end{array}$

5.7.1 Conformal Mapping

5.7.2 Domain Mapping

5.8 Problems

6 Nonlinear Wave Theories

6.1 Introduction

6.2 Classical Stokes: The Method of Successive Approximations

6.3 Traditional Stokes: Lindstedt-Poincare 4th Order Perturbation Solution

6.3.1 Traditional Stokes: Stokes Drift

6.4 Method of Multiple Scales (MMS)

6.5 Stream Function Solutions

6.6 Breaking Progressive Waves 400

6.7 Second-Order Nonlinear Planar Wavemaker Theory 405

6.8 Chaotic Cross Waves: Generalized Melnikov Method (GMM) and Liapunov Exponents

6.9 Problems 
7 Deterministic Dynamics of Small Solid Bodies

7.1 Introduction

7.2 Small Body Hypothesis (Morison Equation )

7.3 Drag $d F_{d}$ and Inertia $d F_{m}$ Forces

7.3.1 Inertia Forces

7.4 Comparison Between a Fixed Cylinder in Accelerating Flow and an Accelerating Cylinder in Still Fluid

7.4.1 Accelerating Cylinder in Still Fluid

7.4.2 Fixed Cylinder in an Accelerating Flow

7.5 Maximum Static-Equivalent Force/Moment (Fixed-Free Beam)

7.6 Parametric Dependency of Force Coefficients $C_{m}$ and $C_{d}$

7.6.1 Relative Importance of $d F_{m}\left(z_{i}, t\right)$ and $d F_{d}\left(z_{i}, t\right)$

7.6.2 Computing the Force Coefficients $C_{m}$ and $C_{d}$

7.6.3 Methods of Analyses

7.6.4 Linearized Drag Force

7.6.5 Laboratory U-Tube Data

7.6.6 Ocean Wave Data

7.7 The Dean Eccentricity Parameter and Data Condition

7.7.1 Dean Error Ellipse and Eccentricity Parameter $E$ (Geometric)

7.7.2 Amplitude/Phase Method (Geometric)

7.7.3 Matrix Condition Numbers (Numerical)

7.8 Modified Wave Force Equation (WFE, Relative Motion Morison Equation)

7.8.1 Articulated Circular Cylindrical Tower: SDOF System

7.8.2 Two Semi-Immersed Horizontal

Circular Cylinders: MDOF System

7.9 Transverse Forces on Bluff Solid Bodies

7.10 Stability of Marine Pipelines

7.11 Problems

8 Deterministic Dynamics of Large Solid Bodies 
8.2 Linearized MDOF Large Solid Body Dynamics 636

8.2.1 Kinematic Body Boundary Conditions (KBBC) 638

8.2.2 Dynamic Body Boundary Conditions (DBBC) 643

8.3 Froude-Kriloff Approximations for Potential Theory 662

8.3.1 Froude-Kriloff Load in 2-D Cartesian Coordinates 663

8.3.2 Froude-Kriloff Load in Circular Cylindrical Coordinates

8.4 Diffraction by a Full-Draft Vertical Circular Cylinder $\quad 672$

8.5 Reciprocity Relationships 682

8.6 Green's Functions and Fredholm Integral Equations 688

8.6.1 Orthonormal Eigenfunction Expansion of Green's Function for 2D Wavemaker

8.7 Wave Loads Computed by the FEM 706

$\begin{array}{lll}8.8 \text { Problems } & 716\end{array}$

9 Real Ocean Waves $\quad 719$

$\begin{array}{lll}9.1 & \text { Introduction } & 719\end{array}$

$\begin{array}{lll}9.2 & \text { Fourier Analyses } & 720\end{array}$

9.3 Ocean Wave Spectra 730

9.3.1 Generic Four-Parameter Wave Density Spectrum 740

9.3.2 Wave and Spectral Parameters Computed from Spectral Moments $m_{n} \quad 747$

9.3.3 Multi-Parameter Theoretical Spectra 752

9.3.4 Spectral Directional Spreading Functions $\quad 754$

9.3.5 Confidence Intervals for FFT Estimates 759

9.4 Probability Functions for Random Waves $\quad 760$

9.4.1 Gaussian (Normal) Probability Distribution 765

9.4.2 Rayleigh Probability Distribution $\quad 772$

9.4.3 Distribution of the Maxima 780

$\begin{array}{lll}9.5 & \text { Wave Groups } & 790\end{array}$

9.5.1 Resolving Incident and Reflected Random Wave Time Series 
9.6 Random Wave Simulations $\quad 808$

9.6.1 Conditional Wave Simulations $\quad 813$

9.7 Data Analyses: An Example from Hurricane CARLA 823

9.8 Random Wave Forces on Small Circular Members 831

9.8.1 Probability Density Function $\mathrm{p}(\mathrm{Y})$ (pdf) and Covariance Function $C_{F_{T} F_{T}}(\tau)$ for Nondeterministic Wave Force per Unit Length for a Small Vertical Circular Pile

9.8.2 Stochastic Response of Space-Frame Offshore Structure

9.9 Frequency Domain Input-Output Transfer Functions 854

9.10 Problems

Bibliography

Author Index

Subject Index 
This page is intentionally left blank 


\section{Preface}

The modest number of topics on waves and wave forces that is reviewed comes primarily from three sources: (1) from four one-quarter in length (10 weeks) graduate courses taught at Oregon State University (OSU) in the USA; viz. (i) linear waves, (ii) nonlinear waves, (iii) random real ocean waves and (iv) wave forces on structures; (2) from Masters and $\mathrm{PhD}$ theses written for the Departments of Civil, Construction and Environmental Engineering and of Mathematics at OSU; and (3) from manuscripts co-authored by the author. Because of these limited resources for the topics reviewed, the topics are intended primarily as a reference for graduate classes on waves and wave loads; although it is hoped that some practicing coastal/ocean engineers may also find these topics of some value. The emphases are (1) on the fundamental physics of the dynamics of fluids and of semi-immersed Lagrangian solid bodies that are responding to wave induced loads; (2) on the scaling of dimensional equations and boundary value problems in order to determine a small dimensionless parameter, $\epsilon$ say, that may be evaluated to linearize the equations and the boundary value problems in order to obtain a linear system; (3) on the replacing of differential and integral calculus equations with algebraic equations that require only algebraic substitutions instead of differentiations and integrations; and (4) on the importance of comparing analytical and numerical computations with data from laboratories and/or nature. More extensive treatments of the topics reviewed here are given from the point of view of naval architecture by Wehausen and Laitone, Surface Waves in Handbook of Physics, Fluid Dynamics III (1960) and from the point of view of coastal and ocean engineering by Mei in The Applied Dynamics of Ocean Surface Waves (1989). 
Chapter 1 introduces the topic of wave forces on coastal and ocean structures and attempts to motivate the reasons for studying the theories that are reviewed.

Chapter 2 reviews the mathematical methods employed in the remaining chapters; and is motivated by the format used by Morse and Ingard, Theoretical Acoustics (1968) Chapter 1.2. The pedagogy used by the author in the four graduate courses cited above is to formulate problems on waves and wave ¿ loads on coastal and ocean structures in generic mathematical nomenclature; . and to then search the mathematical literature for the solutions to the generic mathematical problem formulated rather than associating a generic mathematical solution method to a particular wave or wave load problem on a specific کु. topic or in a specific chapter. By this pedagogy, it is hoped that readers will o look for generic mathematical solutions to wave and wave load problems in the mathematical literature where the solution methods are compactly archived by socific mathematical nomenclature. This will expedite solutions and make all problems on waves and wave loads generic to their mathematical solutions.

Chapter 3 reviews the fundamental laws for the conservation of mass, momentum and energy for an Eulerian fluid field by the differential element method in Cartesian coordinates. The derivations are limited to the differen0 . Eial element method in Cartesian coordinates; but the corresponding results from arbitrary integral control volumes and from tensor analyses are also . of non-uniqueness between the scaling of equations and dimensional analysis are included in order to stress the importance of analyses by both of these methods for numerical and analytical computations and by the subsequent essential comparison between numerical and analytical results with data from 30 laboratories and/or nature.

Chapter 4 begins the surface gravity wave analyses by reviewing longcrested linear wave theory (LWT) applying real-valued elementary transcendental functions. Differential control volumes in Cartesian coordinates are employed for deriving the equations for the boundary value problem for longcrested linear waves and the subsequent solutions for the conservation of mass, momentum and energy flux for both progressive and standing linear waves. Algorithms for computing the propagating eigenvalue (wave number) for the well-posed Sturm-Liouville boundary value problem that is formulated in Chapter 2 are reviewed. 
Chapter 5 reviews a variety of $2 \mathrm{D}$ and $3 \mathrm{D}$ wavemaker theories applying complex-valued elementary transcendental functions. The emphasis of the wavemaker theories reviewed is the application of the wavemaker theory to the radiation potential for computing forces and moments on large semi-immersed Lagrangian solid bodies that are reviewed in Chapter 8 . There is a deliberate and repeated effort to connect this review to the radiation potential for large body forces and moments in Chapter 8 . In addition, integral equations for the coefficients of the eigenfunctions, for the forces and moments on wavemakers and for computing wavemaker power are replaced by algebraic equations that only require algebraic substitutions and eliminate all integral computations. Two-dimensional planar and double-articulated wavemakers are analyzed; and algorithms for computing the evanescent (or "local" wave components, John, 1950 , p. 48) eigenvalues are reviewed. Both amplitude-modulated (AM) and phase-modulated (PM) circular wavemakers are reviewed. Sloshing waves in 2D channels and directional wavemakers in $3 \mathrm{D}$ basins are reviewed. Finally, the mapping of 2D wavemakers by both conformal mappings and by domain mapping are reviewed.

Chapter 6 reviews several nonlinear wave theories. Stokes 2D nonlinear waves are reviewed first by the classical (but extremely tedious) Stokes method of successive approximations and, subsequently, by the traditional Stokes-Lindstedt-Poincare perturbation method. In the traditional StokesLindstedt-Poincare perturbation method there are two methods that may be applied to suppress resonant forcing. One method is to expand the wave celerity $C$ in a perturbation expansion according to $C=\sum_{m=0} \epsilon_{(m+1)}^{m} C$. Another option is to expand the radian wave frequency $\omega$ in a perturbation expansion according to $\omega=\sum_{n=0} \epsilon^{n} \omega_{n}$. Both of these methods are reviewed. Specifically, the first method of expanding the wave celerity $C$ is applied in the traditional Stokes perturbation expansion in Chapter 6.3; and the second method of expanding the radian wave frequency $\omega$ is applied in the nonlinear planar wavemaker theory in Chapter 6.7. In particular, the review of the Stokes-Lindstedt-Poincare traditional perturbation method introduces the method of replacing differential calculus with algebraic substitutions. The numerous repeated term by term differentiations required in the boundary conditions (as many as $\mathbf{7 5}$ differentiations at fourth order!) are replaced by six relatively simple algebraic equations that many times are identically 
equal to zero! The more modern methods of multiple scales (MMS) and the numerical stream function theory are reviewed briefly. Wave breaking is also reviewed briefly. An extension of the $2 \mathrm{D}$ wavemaker theory to weakly nonlinear waves and to Stokes drift in 2D wave channels is included. An analysis of weakly damped cross-waves with surface tension is analyzed by the generalized Melnikov method for nonlinear nonautonomous Hamiltonian systems with contact (canonical) transformations computed by an extension of the Herglotz algorithm to nonautonomous transformations.

Chapter 7 begins the formal analyses of deterministic wave loads on structures by reviewing the dynamics of small Lagrangian solid bodies responding to loads computed from the Morison equation and from the Modified Wave Force equation (relative motion Morison equation). The parametric dependencies of the inertial $C_{m}$ and drag $C_{d}$ force coefficients in the Morison equation are reviewed and are connected to the scaling and dimensional analyses methods reviewed in Chapter 3 . The linearized wave force equation (relative motion Morison equation) is applied to analyze both a single-degree-of-freedom (SDOF) articulated tower and a semi-immersed, three-degree-of-freedom (MDOF) double pontoon system. The linearization of the relative motion wave loads in these two examples makes it possible to separate the wave loads into terms proportional only to the body motions and to terms proportional only to loads on fixed bodies. Because of this linear decomposition of the wave loads, deterministic wave loads on fixed small bodies need not be treated as a separate topic in this review because the wave loads on fixed bodies are identical to the exciting wave loads on dynamically responding small bodies to the first linear approximation! Finally, the chapter concludes by reviewing the transverse lift forces on small bodies and the stability of bottom laid marine pipelines through the surf zone.

Chapter 8 reviews the deterministic dynamics of large Lagrangian solid bodies responding to linear wave loads computed by potential wave theory and the linear progressive wave potentials derived in Chapter 4. Similar to the analyses in Chapter 7, the linear decomposition of the wave loads on large bodies reduces to uncoupled boundary value problems for (1) a scattered wave potential that computes the exciting wave loads on a fixed large Lagrangian body and to (2) a radiated wave potential that computes the restoring or wavemaker loads on an oscillating (wavemaker) body in otherwise still water. This linear decomposition in two separate and uncoupled boundary value problems is the direct consequence of the equalitarian treatment of 
the kinematic and dynamic boundary conditions between the Eulerian fluid field and the large Lagrangian solid body. Because the independent variables of the Eulerian fluid field (viz. space and time) and of the Lagrangian solid body (viz. particle and time) are not the same, the two boundary conditions on the boundary between the Eulerian fluid field and on the Lagrangian solid body must be treated with care. Specifically, the kinematic boundary condition converts the Lagrangian particle velocities of the semi-immersed solid body to Eulerian field velocities by the vector dot product between the time-dependent Lagrangian body velocities and the spatially-dependent unit normal to the Lagrangian solid body. Then the spatial dependencies of the dynamic Eulerian pressure field are integrated around the submerged portions of the semi-immersed Lagrangian solid body so that the wave loads are transformed to only time-dependent Lagrangian variables in the Lagrangian dynamic boundary condition. The boundary value problem for the radiated wave potential is connected repeatedly to the wavemaker theories in Chapter 5 . Froude-Kriloff approximations for wave loads in both Cartesian and circular cylindrical coordinates are reviewed as well as the MacCamy-Fuchs diffraction theory for a full draft vertical cylinder. Reviews of the reciprocity relations and the Green's functions as resolvent kernels in Fredholm integral equations are reviewed. Wave loads computed by the Finite Element Method (FEM) complete the chapter on wave loads on large Lagrangian solid bodies.

Chapter 9 reviews the non-deterministic wave theories and wave loads. Fourier analyses of stochastic processes are reviewed and an application of the finite Fourier transform (FFT) to measured wave data from Hurricane CARLA is given. Generic 4 parameter and multi-parameter wave spectra are reviewed. Gaussian and Rayleigh probability theories are applied to the time series of wave profiles and wave heights, respectively. Wave groups are analyzed by the Hilbert transform and then applied to a stability analysis of coastal rubble-mound breakwater structures with comparisons to laboratory data. This analysis requires an algorithm that is capable of resolving incident and reflected times series (with wave phases as well as wave amplitudes) in order to apply the Hilbert transform and to compute the groupiness of the incident time series. Algorithms for random wave simulations by digital computers for both deterministic spectral amplitude (DSA) and nonderministic spectral amplitude (NSA) random wave simulations are reviewed along with conditional random wave simulations. Random wave forces on a prototype space-frame structure in the Gulf of Mexico are computed by the linearized 
relative motion Morison equation from Chapter 7 applying nondeterministic random waves and are compared with data from Hurricane CARLA on the same space-frame structure. The structural model also includes a dynamic soilspring response algorithm. Finally, the frequency domain transfer functions for Eulerian field variables and for Lagrangian body motions are reviewed.

A Bibliography that contains a relatively large number of references that are not cited explicitly in the main chapters concludes this review.

I have benefitted enormously over the past 30 years from my harmonious collaborations with my colleagues of the College of Engineering, the Department of Mathematics and the College of Oceanic and Atmospheric Sciences at OSU in the USA; from colleagues in laboratories and institutions in Indonesia, Japan, Korea, Poland, Spain and Taiwan; as well as graduate students in both the Department of Civil, Construction and Environmental Engineering and the Department of Mathematics at OSU. In addition, I must also acknowledge my mentors from many academic and governmental institutions that have educated me. Although I feel obligated to cite each of them individually for their contributions to this review; it would require another review of equal length; but I do wish to acknowledge and to express my sincere appreciation individually to each of them for their contributions. However, it is virtually impossible to eliminate all of the theoretical and typographical errors in a review with the number of equations and theories that are reviewed here; and I, alone, bear all of the responsibility for these errors. I have, however, made repeated efforts to proof both the theories and equations; and I apologize here in advance for those errors that have survived all of my scrutiny. In addition, some of the materials that are reviewed such as replacing differential and integral calculus with algebraic substitutions in Chapters 5 and 6 and the applications of the generalized Melnikov method as well as the generalized Herglotz algorithm to analyze chaotic cross-waves in Chapter 6 are relatively new ideas (at least to the author) that are among many others in this review that almost surely require further elaborations and/or clarifications. I solicit these elaborations, clarifications and corrections and I welcome all comments, criticisms, suggestions and corrections. The coastal and ocean waters that cover our planet are wonderful areas to enjoy both for recreation and to benefit from their productivity. May some of the readers of my modest efforts here benefit from these efforts and help all of us to enjoy the beauty and the productivity of our coastal and ocean waters. 